\title{
3) (1) Nivolumab monotherapy in recurrent metastatic urothelial carcinoma (CheckMate 032): a multicentre, open-label, two-stage, multi-arm, phase $1 / 2$ trial
}

\author{
Padmanee Sharma, Margaret K Callahan, Petri Bono, Joseph Kim, Pavlina Spiliopoulou, Emiliano Calvo, Rathi N Pillai, Patrick A Ott, \\ Filippo de Braud, Michael Morse, Dung T Le, Dirk Jaeger, Emily Chan, Chris Harbison, Chen-Sheng Lin, Marina Tschaika, Alex Azrilevich, \\ Jonathan E Rosenberg
}

\section{Summary}

Lancet Oncol 2016; 17: 1590-98 Published Online October 9, 2016 http://dx.doi.org/10.1016/ S1470-2045(16)30496-X

See Comment page 1475

Department of Genitourinary Medical Oncology, Department of Immunology, MD Anderson

Cancer Center, University of Texas, Houston, TX, USA (Prof P Sharma MD); Department of Medicine, Memorial Sloan Kettering Cancer Center, New York, NY, USA (M K Callahan MD, J Rosenberg MD); Department of Oncology, Comprehensive Cancer Center, Helsinki University Hospital and University of Helsinki, Helsinki, Finland (P Bono MD); Prostate and Urologic Cancers Program and Early Drug Development Program, Yale Cancer Center, New Haven, CT, USA (J Kim MD); Department of Medical Oncology, Beatson West of Scotland Cancer Centre, Glasgow, UK (PSpiliopoulou MD); Medical Oncology Division, START Madrid, Centro Integral Oncológico Clara Campal, Madrid, Spain (E Calvo MD); Department of Hematology and Oncology, Emory Winship Cancer Institute, Atlanta, GA, USA (R N Pillai MD); Melanoma Center, Center for Immuno-

Oncology, Dana-Farber Cancer Institute, Boston, MA, USA (PA Ott MD); Department of Medical Oncology, Istituto Nazionale dei

Tumori-Università degli Studi di Milano, Milan, Italy

(Prof F de Braud MD); Duke Cancer Institute, Duke University Medical Center, Durham, NC, USA (Prof M Morse MD); Division of Oncology-Gastrointestinal

Cancer, Sidney Kimmel Comprehensive Cancer Center at Johns Hopkins, Baltimore,

Background Few effective treatments exist for patients with advanced urothelial carcinoma that has progressed after platinum-based chemotherapy. We assessed the activity and safety of nivolumab in patients with locally advanced or metastatic urothelial carcinoma whose disease progressed after previous platinum-based chemotherapy.

Methods In this phase $1 / 2$, multicentre, open-label study, we enrolled patients (age $\geq 18$ years) with urothelial carcinoma of the renal pelvis, ureter, bladder, or urethra at 16 sites in Finland, Germany, Spain, the UK, and the USA. Patients were not selected by PD-L1 expression, but tumour PD-L1 membrane expression was assessed retrospectively. Patients received nivolumab $3 \mathrm{mg} / \mathrm{kg}$ intravenously every 2 weeks until disease progression or treatment discontinuation because of unacceptable toxicity or other protocol-defined reasons, whichever occurred later. The primary endpoint was objective response by investigator assessment. All patients who received at least one dose of the study drug were included in the analyses. We report an interim analysis of this ongoing trial. CheckMate 032 is registered with ClinicalTrials.gov, NCT01928394.

Findings Between June 5, 2014, and April 24, 2015, 86 patients with metastatic urothelial carcinoma were enrolled in the nivolumab monotherapy group and 78 received at least one dose of treatment. At data cutoff (March 24, 2016), the minimum follow-up was 9 months (median 15.2 months, IQR 12.9-16.8). A confirmed investigator-assessed objective response was achieved in $19(24 \cdot 4 \%, 95 \%$ CI 15 -3-35.4) of 78 patients. Grade 3-4 treatment-related adverse events occurred in $17(22 \%)$ of 78 patients; the most common were elevated lipase (four [5\%]), elevated amylase (three [4\%]), and fatigue, maculopapular rash, dyspnoea, decreased lymphocyte count, and decreased neutrophi count (two [3\%] each). Serious adverse events were reported in $36(46 \%)$ of 78 patients and eight (10\%) had a serious adverse event judged to be treatment related. Two (3\%) of 78 patients discontinued because of treatment-related adverse events (grade 4 pneumonitis and grade 4 thrombocytopenia) and subsequently died.

Interpretation Nivolumab monotherapy was associated with a substantial and durable clinical response and a manageable safety profile in previously treated patients with locally advanced or metastatic urothelial carcinoma These data support further investigation of nivolumab monotherapy in advanced urothelial carcinoma.

Funding Bristol-Myers Squibb.

\section{Introduction}

Nearly three decades have passed since the first ground-breaking treatments were developed for patients with metastatic urothelial carcinoma. The combination chemotherapy regimen methotrexate, vinblastine, doxorubicin, and cisplatin has not been surpassed in terms of response and survival. ${ }^{1}$ In 2000 , gemcitabine plus cisplatin was tested as a less toxic alternative, but $37 \%$ of patients could not tolerate the treatment regimen. Decades of research exploring cytotoxic frontline chemotherapies followed, ${ }^{3}$ but no treatments were able to exceed the therapeutic outcomes achieved with the combination of methotrexate, vinblastine, doxorubicin, and cisplatin. About $25-50 \%$ of patients with metastatic urothelial carcinoma are unable to receive cisplatin-based chemotherapy because of renal impairment. ${ }^{4}$ Nonetheless platinum-based combination chemotherapy remains the standard first-line treatment for patients with metastatic urothelial carcinoma. ${ }^{4}$ In the second-line setting, many drugs have been tested, but none have become established as a standard of care because of a low frequency of response (10\% of patients or less). The most intensively studied regimen in the second-line setting-vinflunine plus best supportive care-did not significantly improve overall survival compared with best supportive care in a phase 3 trial (hazard ratio $0 \cdot 9,95 \%$ CI $0 \cdot 7-1 \cdot 1$; intention-to-treat population), ${ }^{5}$ although an increase in median overall survival of 2.6 months was noted with vinflunine in a subsequent analysis of the eligible population that excluded patients with protocol violations at baseline (hazard ratio $0 \cdot 8,95 \%$ CI $0 \cdot 6-1 \cdot 0$; $\mathrm{p}=0 \cdot 023$ ).

Immune checkpoint treatment, consisting of blockade of immune inhibitory pathways, has led to substantial advances in the treatment of cancer. The potential for 
Research in context

\section{Evidence before this study}

We searched PubMed from Dec 1, 1994, to May 31, 2014, using

the search terms "metastatic urothelial carcinoma", "relapsed urothelial carcinoma", "clinical trials", "immune response", "immune checkpoint blockade", and "immunotherapy". Studies identified from the search revealed poor outcomes for patients with recurrent or relapsed urothelial carcinoma, with few treatment options to improve survival. A few papers reported findings from studies investigating immunotherapy in advanced urothelial carcinoma, including BCG treatment. The immune system is a target for treatment in urothelial carcinoma; immunotherapy with BCG is standard treatment for superficial urothelial carcinoma, reducing the risk of local recurrence by about $60 \%$ and leading to 5 -year survival of about $90 \%$ in patients with unifocal disease. Additionally, CD8+ tumour-infiltrating lymphocytes are predictive of survival in muscle-invasive urothelial carcinoma; patients with advanced urothelial cancer and higher numbers of CD8+ tumourinfiltrating lymphocytes within the tumour ( $\geq 8$ cells) seem to have better disease-free and overall survival than those with similar-staged urothelial carcinoma and fewer intra-tumoural CD8+ tumour-infiltrating lymphocytes. Together with the promising anti-PD-L1 data reported in advanced urothelial carcinoma, these findings provided the rationale for further investigation of immune checkpoint blockade with the PD-1 inhibitor nivolumab for recurrent metastatic urothelial carcinoma.

\section{Added value of this study}

This study includes, to our knowledge, the largest datasets for an anti-PD-1 immune checkpoint inhibitor in second-line (and beyond) urothelial carcinoma, a disease for which few treatment options exist. The proportion of patients with an objective response, the duration of responses, and the overall survival reported might signify greater clinical activity than other available options.

\section{Implications of all the available evidence}

Patients with recurrent metastatic urothelial carcinoma have poor clinical prospects because of the scarcity of effective treatments. A growing body of evidence suggests that immune checkpoint inhibition can offer effective treatment for patients with urothelial carcinoma, as in other tumour types. The results presented here support further development of PD-1 checkpoint inhibition in larger trials of this disease.
MD, USA (D T Le MD); Nationa Center for Tumor Diseases, Department of Medical Oncology, Heidelberg University Hospital, Heidelberg, Germany (D Jaeger MD); Department of Medicine, Division of Hematology and Medical Oncology, Vanderbilt-Ingram Cancer Center, Nashville, TN, USA (E Chan MD); and NIVO/IPI LCM Biomarkers (C Harbison PhD), Clinical Biostatistics (C-S Lin PhD), and Global Clinical Research/ Oncology (M Tschaika MD A Azrilevich PharmD) BristolMyers Squibb, Princeton NJ, USA

Correspondence to:

Prof Padmanee Sharma Department of Genitourinary Medical Oncology, Department of Immunology, MD Anderson Cancer Center, The University of Texas, Houston, TX 77030, USA padsharma@mdanderson.org this approach in the treatment of urothelial carcinoma is suggested by the effectiveness of immunotherapy with BCG; given intravesically, BCG induces an immune response against tumour cells and is indicated as adjuvant treatment after surgical resection in patients with high-grade non-muscle-invasive urothelial carcinoma. ${ }^{6}$ The immune checkpoint inhibitor ipilimumab, which blocks CTLA-4, enhanced immune responses and tumour regression in studies of patients with localised urothelial carcinoma. ${ }^{7.8}$

A promising target for immunotherapy is the PD-1/PD-L1 immune checkpoint. PD-1 is expressed on $\mathrm{T}$ cells and can inhibit T-cell responses on interaction with its ligands, PD-L1 and PD-L2; high PD-L1 expression has been found in bladder tumour cells. ${ }^{9,10}$ In a clinical trial with atezolizumab, ${ }^{11}$ an antibody that blocks PD-L1, $15 \%$ of patients with metastatic or surgically unresectable urothelial carcinoma who were previously treated with platinum-based chemotherapy responded to treatment, leading to US Food and Drug Administration approval of this drug for the treatment of patients with locally advanced or metastatic urothelial carcinoma who have disease progression during or after platinum-containing chemotherapy or disease progression within 12 months of neoadjuvant or adjuvant treatment with platinumcontaining chemotherapy.

Treatment with nivolumab, a fully human monoclonal IgG4 antibody that blocks PD-1, has proven effective in several solid tumours. Compared with comparator treatments (eg, dacarbazine, docetaxel, or everolimus), nivolumab improved overall survival in melanoma, ${ }^{12}$ non-small-cell lung cancer, ${ }^{13,14}$ renal-cell carcinoma, ${ }^{15}$ and head and neck cancer, ${ }^{16}$ and studies have shown promising clinical activity in several additional malignancies including Hodgkin's lymphoma ${ }^{17}$ and microsatellite-unstable colorectal cancer. ${ }^{18}$

Nivolumab is being investigated in an ongoing multicentre, open-label, phase $1 / 2$ clinical study of several advanced or metastatic solid tumour types (CheckMate 032). ${ }^{19,20}$ We report the activity and safety of nivolumab monotherapy in a cohort of patients from this study who had locally advanced or metastatic urothelial carcinoma (unselected for PD-L1 expression) and were previously treated with platinum-based chemotherapy.

\section{Methods \\ Study design and participants}

CheckMate 032 is a multicentre, open-label, two-stage, multi-arm, phase $1 / 2$ study. Patients with histologically or cytologically confirmed carcinoma of the renal pelvis, ureter, bladder, or urethra were enrolled at 16 sites in five countries (Finland, Germany, Spain, the UK, and the USA; appendix $\mathrm{p} 3$ ). The protocol is available in the appendix.

The urothelial carcinoma part of this trial consisted of two treatment regimens: the first with nivolumab monotherapy and the second with nivolumab in combination with ipilimumab. The outcomes of patients who received combination treatment with ipilimumab will be reported separately.

Patients aged at least 18 years were eligible if they had progressive disease after at least one previous 
platinum-based chemotherapy treatment for metastatic disease or locally advanced unresectable disease, recurrence within 1 year of completing previous platinumbased neoadjuvant or adjuvant treatment, or had previously refused standard treatment with chemotherapy for the treatment of metastatic (ie, stage IV) or locally advanced unresectable disease. Inclusion criteria were locally advanced or metastatic urothelial carcinoma, an Eastern Cooperative Oncology Group (ECOG) performance status of 0 or 1 , and measurable disease by CT or MRI (per Response Evaluation Criteria in Solid Tumors [RECIST] version 1.1). ${ }^{21}$ Patients were not selected based on tumour PD-L1 expression. Baseline laboratory tests used to assess eligibility were white blood cell counts and measurement of neutrophil, platelet, haemoglobin, serum creatinine, alanine aminotransferase, aspartate aminotransferase, total bilirubin, albumin, lipase, and amylase concentrations. Key exclusion criteria included active brain or leptomeningeal metastases; any serious or uncontrolled medical disorder; history of or active, known, or suspected autoimmune disease (vitiligo, type 1 diabetes mellitus, residual hypothyroidism caused by autoimmune thyroiditis, and disorders not expected to recur in the absence of an external trigger were permitted); need for immunosuppressive doses of systemic corticosteroids ( $>10 \mathrm{mg}$ per day prednisolone equivalents) for at least 2 weeks before study drug treatment; and previous treatment with experimental antitumour vaccines or any modulator of T-cell function or checkpoint pathway. Median survival for patients with relapsed advanced transitional cell carcinoma of the urothelium has been reported as about 4.6-6.9 months. ${ }^{5}$

The study was approved by the institutional review board or independent ethics committee for each centre and was done in accordance with Good Clinical Practice guidelines defined by the International Conference on Harmonisation. All patients provided written informed consent to participate before study participation based on the principles of the Declaration of Helsinki.

\section{Procedures}

Patients with metastatic urothelial carcinoma were enrolled by an interactive voice response system to receive nivolumab $3 \mathrm{mg} / \mathrm{kg}$ intravenously every 2 weeks until progression or treatment discontinuation because of unacceptable toxicity or other protocol-defined reasons, whichever occurred later. Patients in the nivolumab monotherapy group could switch to nivolumab plus ipilimumab (nivolumab $1 \mathrm{mg} / \mathrm{kg}$ and ipilimumab $3 \mathrm{mg} / \mathrm{kg}$ or nivolumab $3 \mathrm{mg} / \mathrm{kg}$ and ipilimumab $1 \mathrm{mg} / \mathrm{kg}$ intravenously, every 3 weeks for four cycles) after progression if they met prespecified criteria, as part of this clinical trial (appendix $p$ 4). Treatment beyond RECIST-defined progression was permitted if nivolumab was tolerated and clinical benefit was noted, on the basis of investigator assessment (appendix p 4). No dose reductions or modifications were permitted. Criteria for dose delay until resolution of a treatment-related adverse event to grade 1 or lower have been described previously. ${ }^{19}$ Adverse events that could lead to a dose delay were grade 2 or worse non-skin events (except for grade 2 fatigue), grade 3 skin events, and grade 3 laboratory abnormalities (except for asymptomatic amylase and lipase increases).

Tumour assessments (CT or MRI, or both) were done by the investigator at baseline (within 28 days before the first dose of study drug), every 6 weeks (within 1 week earlier or later) until week 24 , and every 12 weeks (within 1 week earlier or later) thereafter until disease progression per RECIST. If study treatment was discontinued for reasons other than disease progression, tumour assessments were continued. Laboratory assessments were done within $72 \mathrm{~h}$ before dose until week 24, and within $72 \mathrm{~h}$ before every alternate dose thereafter. Safety assessments were done continuously in all treated patients, and adverse events and laboratory values were graded according to the National Cancer Institute's Common Terminology Criteria for Adverse Events, version 4.0. As described previously, ${ }^{19}$ assessment of tumour PD-L1 protein expression was done retrospectively with a Dako PD-L1 immunohistochemical 28-8 pharmDx kit (Dako North America, Carpinteria, CA, USA) in pretreatment tumour biopsy specimens that were fresh or archived within 3 months before treatment start. Several pretreatment specimens could be tested for PD-L1 expression and used to define PD-L1 status. The most recently collected specimen before the start of study treatment was used to define PD-L1 status, although results were not needed before the start of study treatment. Tumour PD-L1 expression was categorised as positive when staining of tumour-cell membrane, at any intensity, was noted in at least $1 \%$ or at least $5 \%$ of tumour cells in a section that included at least 100 assessable tumour cells in any sample (ie, not necessarily the sample collected closest to the time of study drug treatment). PD-L1 expression thresholds were chosen based on findings from previous studies in other tumour types..$^{12-15}$

\section{Outcomes}

The primary endpoint was the proportion of patients with a confirmed investigator-assessed objective response, defined as the number of patients with a best overall response of complete or partial response per RECIST divided by the number of treated patients. For a complete or partial response to be judged to be a best overall response, the assessment needed to be confirmed by a second scan no less than 4 weeks after the criteria for response was first met. Patients who did not meet response-evaluable criteria (ie, at least one target lesion at baseline and at least one on-study assessment) were judged to be not assessable.

Secondary endpoints were safety, defined as the incidence of treatment-related adverse events leading to drug discontinuation within the first 12 weeks of treatment in patients who had at least one dose of study drug; 
duration of response, defined as the time from partial or complete response until progressive disease or death from any cause; progression-free survival, defined as the time from treatment assignment to the date of the first documented tumour progression or death from any cause, whichever occurred first; and overall survival, defined as the time between the date of treatment assignment and the date of death from any cause.

Objective response, overall survival, and progressionfree survival by PD-L1 expression were exploratory endpoints. In a post-hoc analysis, we also identified patients who had a non-conventional benefit; that is, patients who did not have a best overall response of partial response or complete response before initial RECIST-defined progression, and met at least one of the following criteria: appearance of a new lesion followed by a decrease from baseline of at least $10 \%$ in the sum of the target lesions; initial increase from nadir at least $20 \%$ in the sum of the target lesions followed by reduction from baseline of at least 30\%; and initial increase from nadir at least $20 \%$ in the sum of the target lesions followed by at least two tumour assessments showing no further progression, defined as a $10 \%$ additional increase in the sum of target lesions and new lesions.

\section{Statistical analysis}

We enrolled patients according to a one-stage design, with a total sample size of $60-100$ patients needed to assess whether nivolumab resulted in an objective

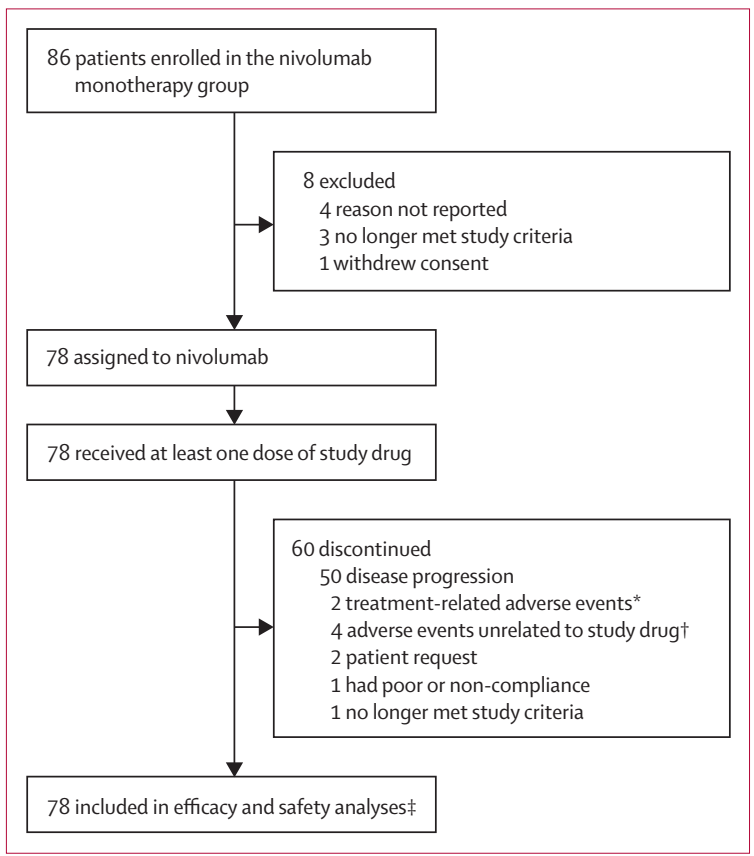

Figure 1: Study profile

*One pneumonitis and one thrombocytopenia. †One each of increased blood creatinine concentration, hepatitis $\mathrm{C}$ infection, anaemia, and urosepsis. $¥$ Not including adverse events in patients who received combination treatments after switching.

\begin{tabular}{|c|c|}
\hline & Nivolumab ( $n=78)$ \\
\hline Age (years) & $65 \cdot 5(31-85)$ \\
\hline$<65$ years & $37(47 \%)$ \\
\hline$\geq 65$ and $<75$ years & $31(40 \%)$ \\
\hline$\geq 75$ years & $10(13 \%)$ \\
\hline \multicolumn{2}{|l|}{ Sex } \\
\hline Male & $54(69 \%)$ \\
\hline Female & $24(31 \%)$ \\
\hline \multicolumn{2}{|l|}{ Race } \\
\hline White & $72(92 \%)$ \\
\hline Black or African-American & $4(5 \%)$ \\
\hline Asian & $1(1 \%)$ \\
\hline Other & $1(1 \%)$ \\
\hline \multicolumn{2}{|l|}{ Smoking status } \\
\hline Present or former & $48(62 \%)$ \\
\hline Never & $29(37 \%)$ \\
\hline Unknown & $1(1 \%)$ \\
\hline \multicolumn{2}{|l|}{ Number of previous regimens } \\
\hline 1 & $26(33 \%)$ \\
\hline $2-3$ & $42(54 \%)$ \\
\hline$>3$ & $10(13 \%)$ \\
\hline \multicolumn{2}{|l|}{ ECOG performance status } \\
\hline 0 & $42(54 \%)$ \\
\hline 1 & $36(46 \%)$ \\
\hline \multicolumn{2}{|l|}{ Baseline metastatic disease } \\
\hline Visceral & $61(78 \%)$ \\
\hline Liver & $20(26 \%)$ \\
\hline Lymph node only & $13(17 \%)$ \\
\hline \multicolumn{2}{|c|}{ Baseline haemoglobin concentration ( $\mathrm{g} / \mathrm{dL})$} \\
\hline$<10$ & $11(14 \%)$ \\
\hline$\geq 10$ & $67(86 \%)$ \\
\hline \multicolumn{2}{|l|}{ Number of Bellmunt risk factors* } \\
\hline 0 & $27(35 \%)$ \\
\hline 1 & $39(50 \%)$ \\
\hline 2 & $8(10 \%)$ \\
\hline 3 & $4(5 \%)$ \\
\hline \multicolumn{2}{|l|}{ Tumour PD-L1 expression } \\
\hline Assessable & $67(86 \%)$ \\
\hline$<1 \%$ & $42(54 \%)$ \\
\hline$\geq 1 \%$ & $25(32 \%)$ \\
\hline$<5 \%$ & $53(68 \%)$ \\
\hline$\geq 5 \%$ & $14(18 \%)$ \\
\hline $\begin{array}{l}\text { Indeterminate, not assessable, } \\
\text { or missing }\end{array}$ & $11(14 \%)$ \\
\hline \multicolumn{2}{|c|}{$\begin{array}{l}\text { Data are median (range) or number (\%). Some percentages do not add up to } \\
100 \text { because of rounding. ECOG=Eastern Cooperative Oncology Group. *ECOG } \\
\text { performance status }>0 \text {, liver metastases, visceral involvement (defined as liver, } \\
\text { lung, bone, or any non-lymph node), lymph-node-only involvement, and } \\
\text { haemoglobin concentration }<10 \mathrm{~g} / \mathrm{dL} \text {. }\end{array}$} \\
\hline
\end{tabular}




\begin{tabular}{|c|c|c|c|}
\hline & $\begin{array}{l}\text { Nivolumab } \\
(n=78)\end{array}$ & $\begin{array}{l}\text { PD-L1 }<1 \% \\
(\mathrm{n}=42)\end{array}$ & $\begin{array}{l}\text { PD-L1 } \geq 1 \% \\
(n=25)\end{array}$ \\
\hline $\begin{array}{l}\text { Confirmed objective } \\
\text { response }\end{array}$ & $\begin{array}{l}19(24 \cdot 4 \% \\
15 \cdot 3-35 \cdot 4)\end{array}$ & $\begin{array}{c}11(26 \cdot 2 \% \\
13 \cdot 9-42 \cdot 0)\end{array}$ & $\begin{array}{l}6(24 \cdot 0 \% \\
9 \cdot 4-45 \cdot 1)\end{array}$ \\
\hline \multicolumn{4}{|l|}{ Best overall response } \\
\hline Complete response & $5(6 \%)$ & $1(2 \%)$ & $4(16 \%)$ \\
\hline Partial response & $14(18 \%)$ & $10(24 \%)$ & $2(8 \%)$ \\
\hline Stable disease & $22(28 \%)$ & $11(26 \%)$ & $8(32 \%)$ \\
\hline Progressive disease & $30(38 \%)$ & $18(43 \%)$ & $8(32 \%)$ \\
\hline Unable to establish & $7(9 \%)$ & $2(5 \%)$ & $3(12 \%)$ \\
\hline \multicolumn{4}{|c|}{$\begin{array}{l}\text { Data are number }(\%, 95 \% \mathrm{Cl}) \text { or number }(\%) \text {. Some percentages do not add up } \\
\text { to } 100 \text { because of rounding. }\end{array}$} \\
\hline
\end{tabular}

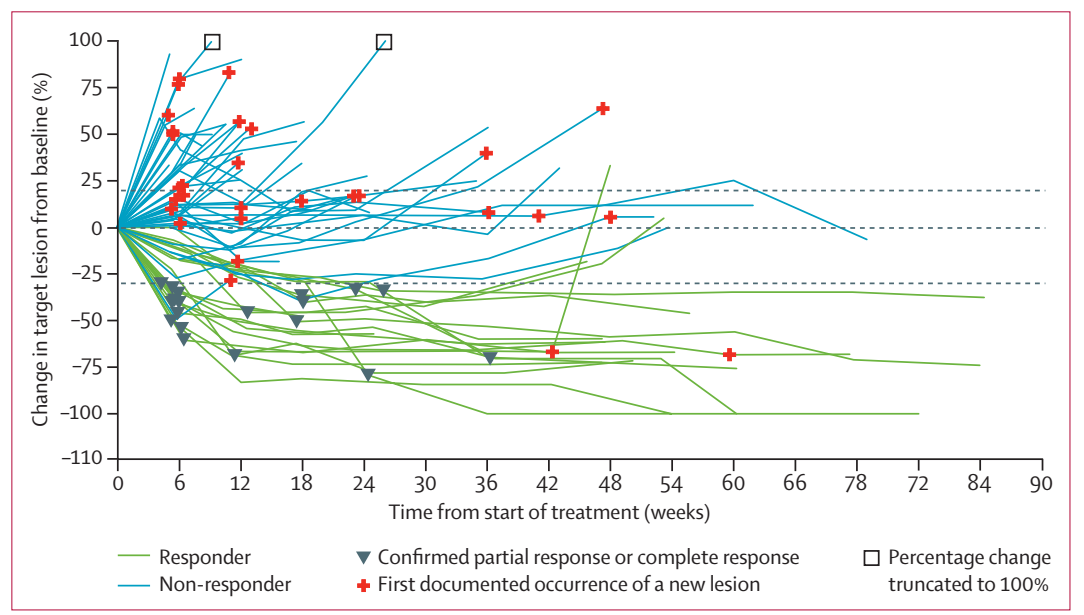

Figure 2: Change in tumour burden over time in 74 response-evaluable patients

Responders were response-evaluable patients with a complete or partial response as best overall response per Response Evaluation Criteria in Solid Tumors version 1.1. Response-evaluable patients were those with a target lesion at baseline and at least one on-treatment tumour assessment. Dotted lines show 30\% decrease, no change, and $20 \%$ increase in size of target lesion from baseline.

response of clinical interest; an objective response $10 \%$ or lower was deemed not to be of clinical value and an objective response of at least $25 \%$ was judged to be of strong clinical interest. The sample size provided $90-97 \%$ power to reject the null hypothesis of a $10 \%$ response rate if the true response rate was $25 \%$, with a two-sided type I error of $5 \%$. Objective response was summarised by a binomial proportion and corresponding two-sided 95\% CIs by the method proposed by Atkinson and Brown. ${ }^{22}$ We summarised the duration of response for patients who achieved confirmed partial response or complete response using the Kaplan-Meier product-limit method, with median values and two-sided 95\% CIs. Tumour shrinkage was characterised by the magnitude of reduction in tumour volume from baseline and was summarised descriptively. Time to response calculations excluded patients whose disease progressed before achieving an objective response. Progression-free survival and overall survival were summarised descriptively using Kaplan-Meier methods, and median values estimated with two-sided $95 \%$ CIs by the Brookmeyer and Crowley method. ${ }^{23}$ To assess the potential effect of switching to combination treatment on overall survival, we did a post-hoc sensitivity analysis of overall survival excluding patients who switched to combination treatment. Progression-free survival and overall survival at 1 year were also estimated, with associated two-sided 95\% CIs calculated with the Greenwood formula. ${ }^{24}$ We summarised response by PD-L1 expression with $95 \%$ CIs using the Clopper-Pearson method. ${ }^{25}$ We also did post-hoc analyses of response according to Bellmunt prognostic risk factors: ECOG performance status, liver metastases, visceral involvement (defined as liver, lung, bone, or any non-lymph node), lymph-node-only involvement, and haemoglobin concentration. We did a post-hoc analysis of immune-mediated adverse events, regardless of causality, that occurred within 100 days of the last dose of nivolumab.

Primary and secondary endpoint analyses included all patients who were treated with nivolumab monotherapy. Objective response and tumour shrinkage were also analysed in the response-evaluable population (ie, those with best overall response in at least one target lesion at baseline assessment and at least one post-baseline assessment). Safety analyses included all patients who were enrolled at least 90 days before database lock. We used SAS version 9.02 for all analyses.

CheckMate 032 is registered with ClinicalTrials.gov, number NCT01928394.

\section{Role of the funding source}

The funder of the study provided the study drug and worked with the investigators to design the study and to collect, analyse, and interpret the data. All drafts of the report were prepared by the corresponding author with input from all co-authors and editorial assistance from professional medical writers, funded by the sponsor. All authors and professional medical writers had full access to all the data in the study and all authors had final responsibility for the decision to submit for publication.

\section{Results}

Between June 5, 2014, and April 24, 2015, 86 patients with metastatic urothelial carcinoma were enrolled in the nivolumab monotherapy group and 78 were treated with nivolumab monotherapy. At the time of data cutoff (March 24, 2016), 60 (77\%) of 78 patients had discontinued treatment, $50(64 \%)$ because of disease progression (figure 1), and 53 (68\%) were continuing to be followed up. $18(23 \%)$ of 78 patients switched to combination treatment with ipilimumab upon disease progression. At data cutoff, minimum follow-up was 9 months (median 15.2 months, IQR $12 \cdot 9-16 \cdot 8)$ and patients had received a median of $8 \cdot 5$ doses (range $1-46$ ) of nivolumab.

Table 1 shows baseline demographics and clinical characteristics of all treated patients. Three patients who 
previously refused standard treatment had not received previous platinum-containing chemotherapy in any setting. 71 (91\%) of 78 patients had tumour tissue samples collected at baseline and PD-L1 expression could be measured in samples from 67 patients; 45 biopsies were obtained from a primary tumour site and 22 from a metastatic site. At least one Bellmunt risk factor (ECOG performance status $>0$, liver metastases, or haemoglobin concentration $<10 \mathrm{~g} / \mathrm{dL}$, visceral involvement, or lymphnode-only involvement) was present in 51 (65\%) of 78 patients.

A confirmed investigator-assessed objective response was achieved in $19(24 \cdot 4 \%, 95 \%$ CI $15 \cdot 3-35 \cdot 4)$ of 78 treated patients, with five patients $(6 \%)$ achieving a complete response and $14(18 \%)$ a partial response (table 2). Four patients were not assessable because they did not have at least one target lesion at baseline and at least one on-study assessment to meet response-evaluable criteria. Of 74 response-evaluable patients, 19 (25.7\%) had a complete or partial response, resulting in an objective response of $25 \cdot 7 \%(95 \%$ CI 16-2-37.2). Change in tumour burden over time for response-evaluable patients is shown in figure 2. 31 (40\%) of 78 patients continued nivolumab monotherapy beyond progression, of whom nine were judged to have a non-conventional benefit (appendix p 4).

At data cutoff, the median duration of response was 9.4 months (IQR 5.7-12.5) and the median time to response was 1.5 months $(1 \cdot 2-4 \cdot 1)$. Of 19 responders, 12 had an ongoing response (11 of whom were still on nivolumab monotherapy), three switched to nivolumab plus ipilimumab combination therapy (one of whom subsequently died), two continued on nivolumab monotherapy after progression, and two died (figure 3).

Median overall survival was 9.7 months $(95 \% \mathrm{CI}$ $7 \cdot 3-16 \cdot 2)$ and $46(59 \%)$ of 78 patients had died at the time of data cutoff (figure 4A). 1-year overall survival was $46 \%$ (95\% CI 34-56). In a post-hoc sensitivity analysis of overall survival excluding patients who switched to combination treatment, 1-year overall survival was $43 \%$ (95\% CI 31-55). Median progression-free survival in the overall treated population was 2.8 months $(95 \%$ CI $1 \cdot 5-5 \cdot 9)$ and 60 (77\%) of 78 patients had disease progression or died by data cutoff (figure 4B). 1-year progression-free survival was 21\% (95\% CI 12-31).

An objective response was achieved in six (24\%, 95\% CI 9-45) of 25 patients with PD-L1 expression of at least 1\%, and in $11(26 \%, 14-42)$ of 42 patients with PD-L1 expression of less than $1 \%$ (table 2). Tumour reduction from baseline in target lesions is shown by PD-L1 expression in the appendix (p 6-7).

Median overall survival was $16 \cdot 2$ months $(95 \%$ CI 7 - 6-not estimable) in patients with PD-L1 expression of at least $1 \%$ and 9.9 months (7.0-not estimable) in those with PD-L1 expression of less than 1\% (appendix p 8). Median progression-free survival was $5 \cdot 5$ months $(95 \%$ CI $1 \cdot 4-11 \cdot 2)$ in patients with PD-L1 expression of at least $1 \%$ and 2.8 months $(1.4-6.5)$ in those with PD-L1 expression of

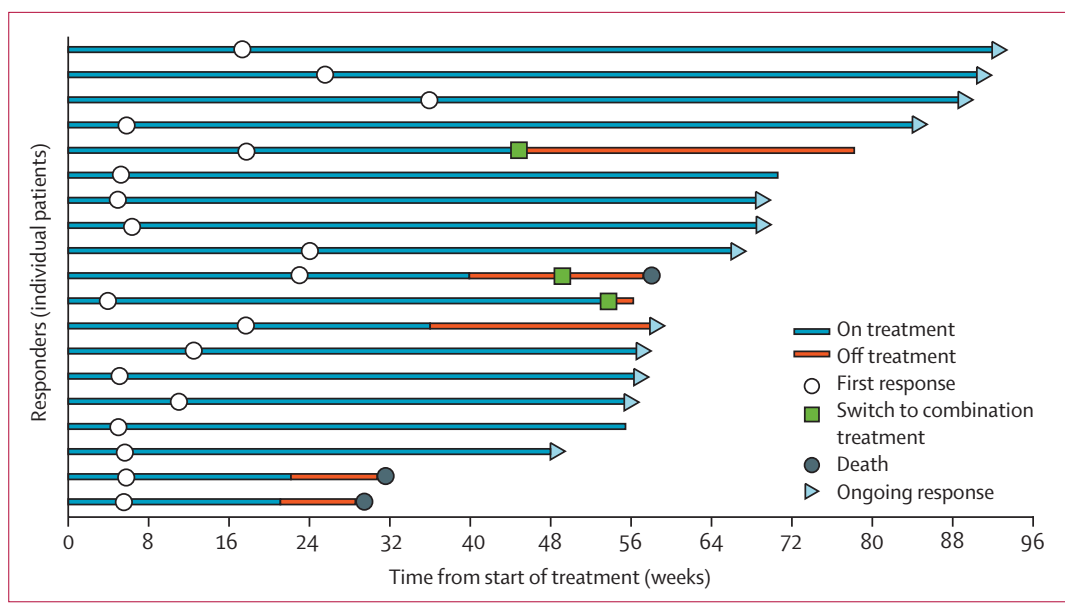

Figure 3: Time to and duration of response

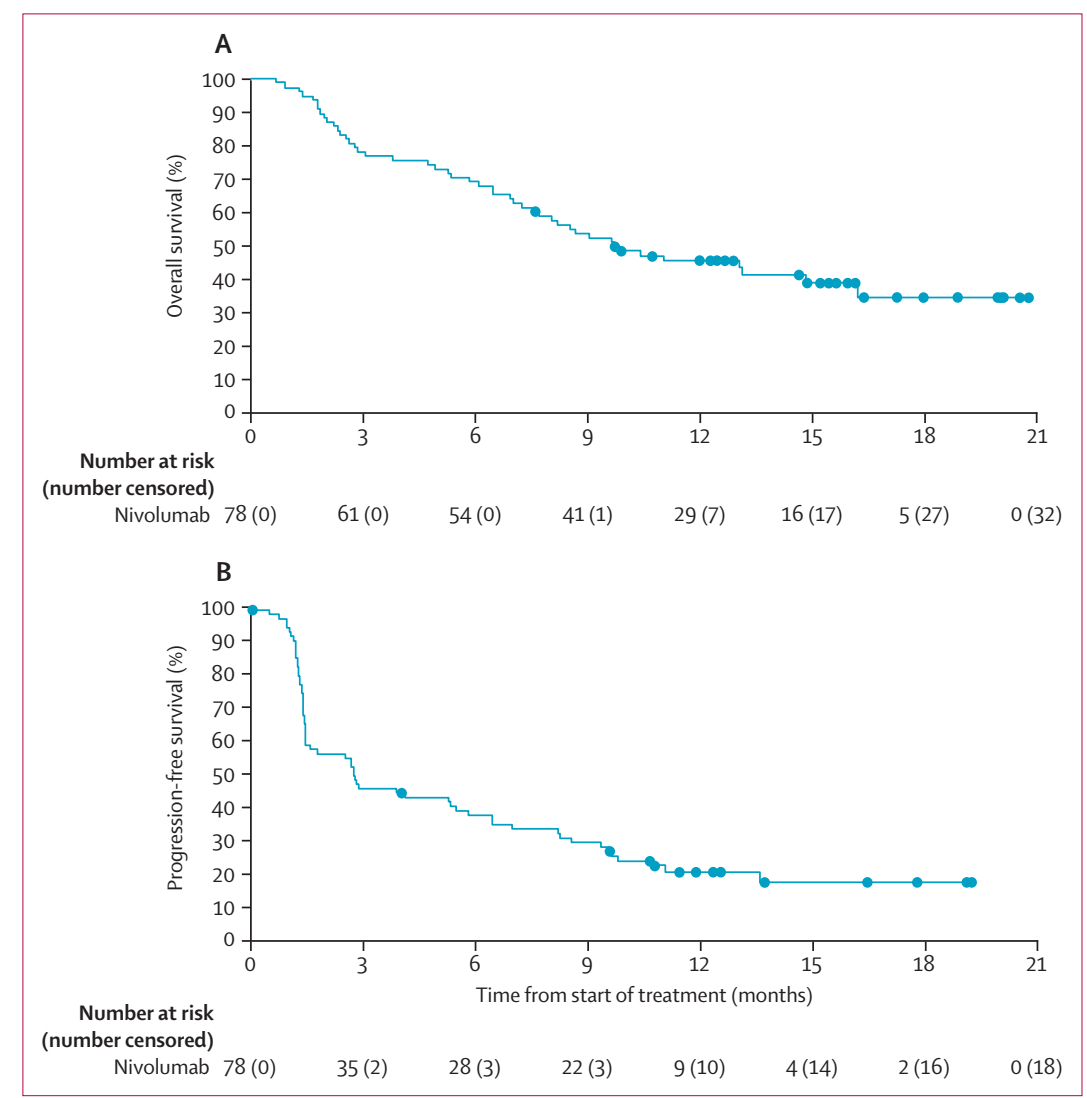

Figure 4: Kaplan-Meier curves of overall survival (A) and progression-free survival (B) Circles are censored patients.

less than $1 \%$ (appendix p 9). Similar results were noted in patients with at least 5\% PD-L1 expression (appendix p 11). The post-hoc analysis of objective response by Bellmunt prognostic risk factors is shown in table 3.

Grade 3 or 4 treatment-related adverse events occurred in $17(22 \%)$ of 78 patients (table 4$)$. The most commonly reported grade 3 or 4 treatment-related adverse events were elevated lipase (four [5\%]), elevated amylase 


\begin{tabular}{|c|c|}
\hline & Number of patients $(\%, 95 \% \mathrm{Cl})(\mathrm{n}=78)$ \\
\hline \multicolumn{2}{|c|}{ ECOG performance status } \\
\hline 0 & $42(26 \cdot 2 \%, 13 \cdot 9-42 \cdot 0)$ \\
\hline 1 & $36(22 \cdot 2 \%, 10 \cdot 1-39 \cdot 2)$ \\
\hline \multicolumn{2}{|c|}{ Liver metastasis } \\
\hline Yes & $20(15 \cdot 0 \%, 3 \cdot 2-37 \cdot 9)$ \\
\hline No & $58(27 \cdot 6 \%, 16 \cdot 7-40 \cdot 9)$ \\
\hline \multicolumn{2}{|c|}{ Visceral metastasis } \\
\hline Yes & $66(21 \cdot 2 \%, 12 \cdot 1-33 \cdot 0)$ \\
\hline No & $12(41 \cdot 7 \%, 15 \cdot 2-72 \cdot 3)$ \\
\hline \multicolumn{2}{|c|}{ Lymph node only } \\
\hline Yes & $11(36 \cdot 4 \%, 10 \cdot 9-69 \cdot 2)$ \\
\hline No & $67(22 \cdot 4 \%, 13 \cdot 1-34 \cdot 2)$ \\
\hline \multicolumn{2}{|c|}{ Haemoglobin concentration $<10 \mathrm{~g} / \mathrm{dL}$} \\
\hline Yes & $11(18 \cdot 2 \%, 2 \cdot 3-51 \cdot 8)$ \\
\hline No & $67(25 \cdot 4 \%, 15 \cdot 5-37 \cdot 5)$ \\
\hline \multicolumn{2}{|c|}{ ECOG=Eastern Cooperative Oncology Group. } \\
\hline
\end{tabular}

(three [4\%]), and fatigue, maculopapular rash, dyspnoea, decreased lymphocyte count, and decreased neutrophil count (two [3\%] each). Treatment-related adverse events of special interest potentially associated with the use of nivolumab of any grade were skin (33 [42\%]), gastrointestinal (eight $[10 \%]$ ), renal (seven [9\%]), hepatic (four [5\%]), and pulmonary adverse events (two [3\%]). A post-hoc analysis of immune-mediated adverse events, regardless of causality, that occurred within 100 days of the last dose of nivolumab is shown in the appendix ( $\mathrm{p} 10)$.

Serious adverse events were reported in 36 (46\%) of 78 patients. Ten serious adverse events judged to be treatment related occurred in eight (10\%) of 78 patients: colitis (grade 3-4), diarrhoea (grade 1-2), mouth ulceration (grade 1-2), nausea (grade 3-4), oral pain (grade 1-2), thrombocytopenia (patient died), fatigue (grade 3-4), hyponatraemia (grade 3-4), acute kidney injury (grade 3-4), and pneumonitis (patient died).

Two (3\%) of 78 patients discontinued treatment because of treatment-related adverse events (grade 4 pneumonitis and grade 4 thrombocytopenia). Both events were fatal and are described in the appendix ( $p 5$ ). 31 patients died of disease progression. Other than disease progression and treatment-related adverse events as causes of death, the following additional deaths were reported: three (4\%) from unknown causes, and one $(1 \%)$ due to sepsis that was not deemed related to study drug. Adverse events leading to treatment discontinuation but not deemed related to study drug were reported in four patients: one patient each had increased blood creatinine (grade 2), hepatitis $\mathrm{C}$ infection (grade 3 ), anaemia (grade 3), and urosepsis (grade 3). 28 (36\%) of 78 patients had at least one dose delay; 52 doses were delayed from a total of 982 doses received (5\%), 34 (65\%) of which were delayed because of adverse events.

\begin{tabular}{|c|c|c|c|}
\hline & Grade 1-2 & Grade 3 & Grade 4 \\
\hline Any event & $46(59 \%)$ & $17(22 \%)$ & 0 \\
\hline Fatigue & $26(33 \%)$ & $2(3 \%)$ & 0 \\
\hline Pruritus & $23(29 \%)$ & 0 & 0 \\
\hline Rash, maculopapular & $12(15 \%)$ & $2(3 \%)$ & 0 \\
\hline Lipase elevated & $7(9 \%)$ & $4(5 \%)$ & 0 \\
\hline Nausea & $9(12 \%)$ & $1(1 \%)$ & 0 \\
\hline Arthralgia & $9(12 \%)$ & 0 & 0 \\
\hline Anaemia & $8(10 \%)$ & 0 & 0 \\
\hline Amylase increased & $4(5 \%)$ & $3(4 \%)$ & 0 \\
\hline Dyspnoea & $4(5 \%)$ & $1(1 \%)$ & $1(1 \%)^{*}$ \\
\hline $\begin{array}{l}\text { Lymphocyte count } \\
\text { decreased }\end{array}$ & $3(4 \%)$ & $2(3 \%)$ & 0 \\
\hline Hyperglycaemia & $4(5 \%)$ & $1(1 \%)$ & 0 \\
\hline $\begin{array}{l}\text { Neutrophil count } \\
\text { decreased }\end{array}$ & $1(1 \%)$ & $2(3 \%)$ & 0 \\
\hline $\begin{array}{l}\text { White blood cell count } \\
\text { decreased }\end{array}$ & $2(3 \%)$ & $1(1 \%)$ & 0 \\
\hline Hyponatraemia & $1(1 \%)$ & $1(1 \%)$ & 0 \\
\hline Dermatitis acneiform & $1(1 \%)$ & $1(1 \%)$ & 0 \\
\hline Wheezing & $1(1 \%)$ & $1(1 \%)$ & 0 \\
\hline Acute kidney injury & 0 & $1(1 \%)$ & 0 \\
\hline $\begin{array}{l}\text { Aspartate } \\
\text { aminotransferase } \\
\text { increased }\end{array}$ & 0 & $1(1 \%)$ & 0 \\
\hline Back pain & 0 & $1(1 \%)$ & 0 \\
\hline Colitis & 0 & $1(1 \%)$ & 0 \\
\hline \multicolumn{4}{|c|}{$\begin{array}{l}\text { Data are number of patients (\%). Adverse events that occurred after patients } \\
\text { crossed over from nivolumab } 3 \mathrm{mg} / \mathrm{kg} \text { to combination treatment were excluded. } \\
\text { Some patients had more than one adverse event. Two patients had a } \\
\text { treatment-related event that led to death (one case of thrombocytopenia and one } \\
\text { case of pneumonitis); both cases are described in greater detail in the } \\
\text { appendix (p5). }{ }^{*} \text { This patient subsequently died from pneumonitis and was } \\
\text { counted in the grade } 5 \text { rather than the grade } 4 \text { total as a treatment-related death. }\end{array}$} \\
\hline \multicolumn{4}{|c|}{$\begin{array}{l}\text { Table 4: Treatment-related adverse events of any grade reported in at } \\
\text { least } 10 \% \text { of patients and all grade } 3-4 \text { events in } 78 \text { patients who } \\
\text { received nivolumab }\end{array}$} \\
\hline
\end{tabular}

\section{Discussion}

In this study, nivolumab monotherapy was associated with a substantial and durable tumour response, promising survival, and acceptable safety in patients with locally advanced or metastatic urothelial carcinoma who were previously treated with platinum-based chemotherapy. Objective response was not dependent on tumour PD-L1 expression and was consistent across patient subgroups on the basis of key prognostic factors.

Patients with metastatic urothelial carcinoma who have progressive or relapsed disease after platinum-based combination chemotherapy have poor prognosis, with a median survival of about 6 months. Several chemotherapies tested in the second-line setting have not shown significant activity and have been associated with substantial toxicity. ${ }^{26-28}$ Immune checkpoint treatments have shown substantial clinical responses across several tumour types, including urothelial carcinoma. ${ }^{11-18,29,30}$ The results reported here 
compare favourably with outcomes reported in a recent phase 2 clinical trial in urothelial carcinoma with the anti-PD-L1 antibody atezolizumab. ${ }^{11}$ The proportion of patients who achieved an objective response in our study seemed consistent across different prognostic risk factor subgroups, although sample sizes were small in these analyses, and in the post-hoc sensitivity analysis of overall survival excluding patients who switched to combination treatment, there was no effect on 1-year survival.

Nivolumab was also well tolerated in this population; the adverse-event profile compares favourably with previously tested chemotherapies and no new safety signals were noted compared with nivolumab studies across a wide range of tumour types. ${ }^{12-15,17}$ Additionally, in this cohort of patients who tend to have several comorbidities and impaired renal function, nivolumab was tolerated over several doses, suggesting a manageable tolerability profile in the longer term. Two patients discontinued treatment because of treatment-related adverse events (pneumonitis and thrombocytopenia); both events were subsequently fatal.

PD-L1 expression on tumour cells, as defined by the Dako immunohistochemical assay, was not associated with objective responses; patients whose tumours were defined as having at least $1 \%$ of tumour cells expressing PD-L1 achieved an objective response with a similar frequency as those whose tumours had less than $1 \%$ of tumour cells expressing PD-L1. Patients whose tumours had at least $1 \%$ of tumour cells expressing PD-L1 had a median overall survival of over 16 months, whereas those whose tumours had less than $1 \%$ of tumour cells expressing PD-L1 had a median overall survival of almost 10 months. Longer follow-up is needed to clarify whether this difference in median overall survival translates into long-term differences. Inter-tumour heterogeneity, both between primary and metastatic lesions and between different metastatic lesions, could have contributed to a false-negative PD-L1 result, although this is mitigated by the fact that patients were classified as having positive PD-L1 expression if they had any positive sample (ie, not necessarily the sample collected closest to the time of study drug treatment). Establishing whether these data are reproducible in a larger cohort of patients is important, and further insights will be provided by an ongoing phase 2 study (CheckMate 275; NCT02387996). In view of recent data suggesting the prognostic value of PD-L1 immune cell (but not PD-L1 tumour cell) expression as a biomarker of response to atezolizumab in patients with metastatic urothelial carcinoma, ${ }^{11}$ further investigation of tumour response with nivolumab according to immune cell PD-L1 expression is warranted.

Limitations of the study include the absence of a standard current practice comparator because of the scarcity of effective treatments in this setting, the small sample size, and the short follow-up period, which precluded further insights into the effect of nivolumab on long-term survival. Also, data on the efficacy and safety of treatment beyond progression, as well as protein expression scoring, were not available, but are being analysed and will be reported at a later date.

In summary, nivolumab monotherapy was active and safe in previously treated patients with locally advanced or metastatic urothelial carcinoma. These data suggest that this immune checkpoint therapy has activity in a patient population with limited treatment options.

\section{Contributors}

PSh and MKC conceived and designed the study. PSh, MKC, PB, JK, PSp, ECa, RNP, PAO, FdB, MM, DTL, DJ, ECh, and JER collected and assembled data. All authors analysed and interpreted data, and wrote and revised the manuscript.

\section{Declaration of interests}

PSh has received fees for advisory board participation for Jounce and Kite; fees for consultancy from Jounce, Kite, Bristol-Myers Squibb, AstraZeneca, and Amgen; and stock or stock options from Jounce and Kite. MKC has received grants from Bristol-Myers Squibb; consultancy fees from AstraZeneca and Moderna; and payment for lectures from Clinical Care Options. PB has received honoraria from Bristol-Myers Squibb, Pfizer, MSD, and Orion Pharma, and research funding from Novartis. RNP has received a travel grant from Bristol-Myers Squibb for an investigator meeting. PAO has received a grant from BristolMyers Squibb and consultancy fees from Bristol-Myers Squibb, Amgen, Celldex, Alexion, and Cytomx. FdB has received consultancy fees from Tiziana Life Sciences, Bristol-Myers Squibb, MSD, Servier, Eli Lilly, Merck Serono, GlaxoSmithKline, and Novartis, and speaker fees from Bristol-Myers Squibb, Eli Lilly, Roche, and ACCMED. MM has received consultancy fees from Etubics and Boehringer Ingelheim, and speaker fees from Genentech, Novartis, Sanofi, Regeneron, Lexicon, Ipsen, Onyx, Bayer, Taiho, Merrimack, and Celgene. DJ and AA are employees of Bristol-Myers Squibb. ECh has received grants from Bristol-Myers Squibb and consultancy fees for advisory board participation from EMD Serono, Taiho, Bayer, Advaxis, Amgen, Lilly, and Castle Biosciences. CH, C-SL, and MT are employees of and hold stock options with Bristol-Myers Squibb. JER has received grants from Bristol-Myers Squibb, Novartis, and Roche/Genentech; has received consultancy fees from Roche/Genentech, AstraZeneca, Eli Lilly, Agensys, Sanofi US Services, Oncogenex, Onyx, Dendreon, Bristol-Myers Squibb, and Boehringer Ingelheim; and holds stock or stock options with Illumina and Merck. JK, PSp, ECa, and DTL declare no competing interests.

\section{Acknowledgments}

We thank the patients and their families, as well as the participating study teams, for making this study possible; the staff of Dako North America for collaborative development of the automated immunohistochemical assay for PD-L1 assessment; and Michael Cunningham for serving as the protocol manager. This research was supported in part through the NIH/NCI Cancer Center Support Grant P30 CA008748. Medical writing and editorial assistance was funded by Bristol-Myers Squibb and provided by Rachel Mason, Tom Rees, and Lawrence Hargett of PPSI (a PAREXEL company).

\section{References}

1 Logothetis CJ, Dexeus FH, Finn L, et al. A prospective randomized trial comparing MVAC and CISCA chemotherapy for patients with metastatic urothelial tumors. J Clin Oncol 1990; 8: 1050-55.

2 von der Maase H, Hansen SW, Roberts JT, et al. Gemcitabine and cisplatin versus methotrexate, vinblastine, doxorubicin, and cisplatin in advanced or metastatic bladder cancer: results of a large, randomized, multinational, multicenter, phase III study. J Clin Oncol 2000; 18: 3068-77.

3 Bellmunt J, von der Maase H, Mead GM, et al. Randomized phase III study comparing paclitaxel/cisplatin/gemcitabine and gemcitabine/cisplatin in patients with locally advanced or metastatic urothelial cancer without prior systemic therapy: EORTC Intergroup Study 30987. J Clin Oncol 2012; 30: 1107-13. 
4 Dash A, Galsky MD, Vickers AJ, et al. Impact of renal impairment on eligibility for adjuvant cisplatin-based chemotherapy in patients with urothelial carcinoma of the bladder. Cancer 2006; 107: 506-13.

5 Bellmunt J, Fougeray R, Rosenberg JE, et al. Long-term survival results of a randomized phase III trial of vinflunine plus best supportive care versus best supportive care alone in advanced urothelial carcinoma patients after failure of platinum-based chemotherapy. Ann Oncol 2013; 24: 1466-72.

6 Babjuk M, Burger M, Zigeuner R, et al. EAU guidelines on non-muscle-invasive urothelial carcinoma of the bladder: update 2013. Eur Urol 2013; 64: 639-53.

7 Liakou CI, Kamat A, Tang DN, et al. CTLA-4 blockade increases IFNg-producing CD4+ICOShi cells to shift the ratio of effector to regulatory T cells in cancer patients. Proc Natl Acad Sci USA 2008 105: 14987-92.

8 Carthon BC, Wolchok JD, Yuan J, et al. Preoperative CTLA-4 blockade: tolerability and immune monitoring in the setting of a presurgical clinical trial. Clin Cancer Res 2010; 16: 2861-71.

9 Faraj SF, Munari E, Guner G, et al. Assessment of tumoral PD-L1 expression and intratumoral CD8+ T cells in urothelial carcinoma. Urology 2015; 85: 703-06.

10 Inman BA, Sebo TJ, Frigola X, et al. PD-L1 (B7-H1) expression by urothelial carcinoma of the bladder and BCG-induced granulomata: associations with localized stage progression. Cancer 2007; 109: 1499-505.

11 Rosenberg JE, Hoffman-Censits J, Powles T, et al. Atezolizumab in patients with locally advanced and metastatic urothelial carcinoma who have progressed following treatment with platinum-based chemotherapy: a single-arm, multicentre, phase 2 trial. Lancet 2016; 387: 1909-20.

12 Robert C, Long GV, Brady B, et al. Nivolumab in previously untreated melanoma without BRAF mutation. N Engl J Med 2015; 372: 320-30.

13 Borghaei H, Paz-Ares L, Horn L, et al. Nivolumab versus docetaxel in advanced nonsquamous non-small-cell lung cancer. $N$ Engl J Med 2015; 373: 1627-39.

14 Brahmer J, Reckamp KL, Baas P, et al. Nivolumab versus docetaxel in advanced squamous-cell non-small-cell lung cancer. $N$ Engl J Med 2015; 373: 123-35.

15 Motzer RJ, Escudier B, McDermott DF, et al. Nivolumab versus everolimus in advanced renal-cell carcinoma. N Engl J Med 2015; 373: $1803-13$.

16 Nivolumab doubles survival for patients with HNSCC. Cancer Discov 2016; 6: OF3.

17 Ansell SM, Lesokhin AM, Borrello I, et al. PD-1 blockade with nivolumab in relapsed or refractory Hodgkin's lymphoma. N Engl J Med 2015; 372: 311-19.
18 Overman MJ, Kopetz S, McDermott RS, et al. Nivolumab \pm ipilimumab in treatment (tx) of patients (pts) with metastatic colorectal cancer (mCRC) with and without high microsatellite instability (MSI-H): CheckMate-142 interim results. Proc Am Soc Clin Oncol 2016; 34 (15 suppl): 3501 (abstr).

19 Antonia SJ, López-Martin JA, Bendell J, et al. Nivolumab alone and nivolumab plus ipilimumab in recurrent small-cell lung cancer (CheckMate 032): a multicentre, open-label, phase 1/2 trial. Lancet Oncol 2016; 17: 883-95.

20 Le DT, Bendell JC, Calvo E, et al. Safety and activity of nivolumab monotherapy in advanced and metastatic $(\mathrm{A} / \mathrm{M})$ gastric or gastroesophageal junction cancer (GC/GEC): results from the CheckMate-032 study. Proc Am Soc Clin Oncol 2016; 34 (4 suppl): 6 (abstr).

21 Eisenhauer EA, Therasse P, Bogaerts J, et al. New Response Evaluation Criteria in Solid Tumours: revised RECIST guideline (version 1.1). Eur J Cancer 2009; 45: 228-47.

22 Atkinson EN, Brown BW. Confidence limits for probability of response in multistage phase II clinical trials. Biometrics 1985; 41: 741-44.

23 Brookmeyer R, Crowley J. A confidence interval for the median survival time. Biometrics 1982; 38: 29-41.

24 Greenwood M. The natural duration of cancer. Rep Public Health Med Subj 1926; 33: 1-26.

25 Clopper CJ, Pearson ES. The use of confidence or fiducial limits illustrated in the case of the binomial. Biometrika 1934; 26: 404-13.

26 Choueiri TK, Ross RW, Jacobus S, et al. Double-blind, randomized trial of docetaxel plus vandetanib versus docetaxel plus placebo in platinum-pretreated metastatic urothelial cancer. J Clin Oncol 2012; 30: $507-12$.

27 Bambury RM, Benjamin DJ, Chaim JL, et al. The safety and efficacy of single-agent pemetrexed in platinum-resistant advanced urothelial carcinoma: a large single-institution experience. Oncologist 2015; 20: 508-15.

28 Hainsworth JD, Meluch AA, Litchy S, et al. Paclitaxel, carboplatin and gemcitabine in the treatment of patients with advanced transitional cell carcinoma of the urothelium. Cancer 2005 103: 2298-303.

29 Sharma P, Allison JP. Immune checkpoint targeting in cancer therapy: toward combination strategies with curative potential. Cell 2015; 161: 205-14.

30 Sharma P, Allison JP. The future of immune checkpoint therapy. Science 2015; 348: 56-61. 
Reproduced with permission of the copyright owner. Further reproduction prohibited without permission. 